\title{
Claudin-7 modulates cell-matrix adhesion that controls cell migration, invasion and attachment of human HCC827 lung cancer cells
}

\author{
DO HYUNG KIM ${ }^{1}$, QUN LU ${ }^{1,2}$ and YAN-HUA CHEN ${ }^{1,2}$ \\ ${ }^{1}$ Department of Anatomy and Cell Biology, ${ }^{2}$ Leo Jenkins Cancer Center, Brody School of Medicine, \\ East Carolina University, Greenville, NC 27834, USA
}

Received August 7, 2018; Accepted December 31, 2018

DOI: $10.3892 / 01.2019 .9909$

\begin{abstract}
Claudins are a family of tight junction proteins, and serve important roles in epithelial barrier, selective ion transports and cancer metastasis. Although the exact role of claudin-7 in human lung cancer has not been completely elucidated, recent clinical studies have demonstrated that claudin-7 is associated with the survival of patients with lung cancer. Our previous studies have demonstrated that claudin-7 forms a protein complex with integrin $\beta 1$ in human lung cancer cells. The knockdown (KD) of claudin-7 by short hairpin RNA (shRNA) reduced integrin $\beta 1$ expression and increased the cell proliferative rate, whereas claudin-7 re-expression in the $\mathrm{KD}$ cells decreased the cell proliferation. It is unknown as to whether claudin-7 and integrin $\beta 1$ regulate cell proliferation and invasion synergistically or independently. In the present study, it was observed that ectopic expression of integrin $\beta 1$ in claudin-7 KD lung cancer cells did not reduce the cell proliferation. However, integrin $\beta 1$-transfected cells migrated more effectively in wound healing and cell invasion assays and were more adhesive in a cell attachment assay when compared with those of claudin-7 KD cells. This indicates that claudin-7 controls cell proliferation, while cell attachment and motility were regulated partially through integrin $\beta 1$. Additionally, claudin-7 overexpression in claudin-7 KD cells resulted in an improved ability to attach to the surface of cell culture plates and a higher expression of focal adhesion proteins when compared with claudin-7 non-KD control cells, which supports the role of claudin-7 in cell adhesion and motility. Taken together, these data suggest that claudin-7 regulates cell motility through integrin $\beta 1$, providing additional insight into the roles of claudins in carcinogenesis and cancer cell metastasis.
\end{abstract}

Correspondence to: Dr Yan-Hua Chen, Department of Anatomy and Cell Biology, Brody School of Medicine, East Carolina University, 600 Moye Boulevard, Greenville, NC 27834, USA

E-mail: cheny@ecu.edu

Key words: tight junctions, claudin-7, cell migration, cell invasion, cell attachment, human lung cancer cells

\section{Introduction}

Human lung cancer is the second leading cause of mortality in the United States (1). The lung expresses various tight junction (TJ) proteins depending on their compartments, including claudin-1, $-2,-3,-5,-7,-8$, and -18 (2). TJ proteins are one of the cellular junctional proteins located at the apical side of epithelial cells, and they regulate paracellular permeability between neighboring epithelial cells (3). One of the TJ proteins is the claudin family that consists of four transmembrane spanning proteins (3). Although the function of claudins as epithelial barriers for the maintenance of cell polarity and selective ion transport has been well established (4), their role in diseases, including cancer, is unclear. However, a possible link between TJs and metastasis has been recently demonstrated using human colon cancer cell lines in vitro $(5,6)$. The varying levels of claudin expression may be correlated to cancer progression (7). Additionally, claudin-5 has been shown to form a protein complex with ROCK and N-WASP and promote actin cytoskeletal movement in breast cancer cells (8), suggesting that $\mathrm{TJ}$ proteins are crucial for cancer cell motility.

A recent clinical research study has shown that claudin-7 expression is associated with the survival of lung cancer patients after surgery (9), suggesting the role of claudin-7 in cancer progression. Results from our previous study demonstrated that claudin-7 knockdown (KD) in HCC827 human lung cancer cell lines increased cell proliferation and reduced integrin $\beta 1$ expression and cell adhesion (10). Interestingly, claudin-7 was able to form a protein complex with integrin $\beta 1$ and was partially co-localized at the basolateral membrane of HCC827 control cells (10). This suggests a possibility that claudin- 7 and integrin $\beta 1$ co-regulate cellular events, including cell proliferation and adhesion; however, this has not been fully explored. Several studies have shown the basal localization of claudin-7 in the epithelial cells of several organs, including mammary gland, kidney, and uterine, suggesting the roles of claudin-7 in cell-matrix adhesion (11-13) and vesicle trafficking (13). In this study, we investigated whether integrin $\beta 1$ and claudin-7 independently or synergistically functioned on cell proliferation, adhesion, migration, invasion, and attachment. Our results demonstrate that ectopic expression of integrin $\beta 1$ partially recovers the cell adhesion, migration, invasion and attachment, but not cell proliferation, of claudin-7 KD cells. 


\section{Materials and methods}

Antibodies. Rabbit polyclonal anti-phospho-Y397-FAK, anti-FAK, anti-phospho-Y118-Paxillin, and anti-GAPDH were purchased from Cell Signaling Technology (Danvers, MA, USA). Anti-integrin $\beta 1$ antibodies were obtained from BD Biosciences and Santa Cruz Biotechnology, Inc. (Dallas, TX, USA). Anti-Paxillin antibody was from BD Transduction Laboratories (San Jose, CA, USA). The secondary anti-mouse and anti-rabbit antibodies tagged with HRP were purchased from Promega (Madison, WI, USA). Rabbit polyclonal anti-claudin-7 antibody was obtained from Immuno-Biological Laboratories (Gunma, Japan), and mouse monoclonal anti-Myc antibody was obtained from Thermo Fisher Scientific, Inc. (Waltham, MA, USA).

Cell lines and reagents. The HCC 827 human non-small cell lung cancer (NSCLC) cell line was obtained from American Type Culture Collection (ATCC) (Manassas, VA, USA) and cultured in RPMI-1640 medium (Thermo Fisher Scientific, Inc.) supplemented with heat-inactivated $10 \%$ fetal bovine serum (HyClone; GE Healthcare, Chicago, IL, USA), $1 \%$ $10,000 \mathrm{U} / \mathrm{ml}$ penicillin, and $10,000 \mu \mathrm{g} / \mathrm{ml}$ streptomycin in a $37^{\circ} \mathrm{C}, 5 \% \mathrm{CO}_{2}$ humidified incubator. $\mathrm{HCC} 827$ control or Claudin-7 KD cell lines were previously established (10).

Transfection and establishment of stably transfected cell lines. In order to establish the stable transfection of integrin $\beta 1$ in HCC827 KD cells (KD+b1 cells), the integrin $\beta 1$ cDNA vector (Transomics, Huntsville, AL, USA) was digested at EcoRI and NotI restriction enzyme sites. The size of integrin $\beta 1$ cDNA insert was confirmed from DNA electrophoresis. The insert was gel-purified using a Gel Extraction kit (Qiagen, Inc., Valencia, CA, USA), and then sub-cloned to a pcDNA3.1 vector at EcoRI and NotI restriction sites. After the pcDNA3.1-integrin $\beta 1 \mathrm{cDNA}$ vector was transfected to HCC 827 KD cells using Amaxa Nucleofector ${ }^{\mathrm{TM}} \mathrm{Kit} \mathrm{V}$ reagent (Lonza, South Plainfield, NJ, USA) by electroporation, the stably transfected cells were selected at $600 \mu \mathrm{g} / \mathrm{ml}$ Geneticin (G418) for 4 weeks. The stable transfectants were maintained in the culture medium containing $300 \mu \mathrm{g} / \mathrm{ml} \mathrm{G} 418$. For the transient transfection, pcDNA3.1-claudin-7-myc (mouse claudin-7 cDNA) vector was transfected to HCC827 KD cell lines and the transfectants were incubated and recovered overnight under an antibiotics-free medium. The transfectants were given fresh media the next day and used for the experiment within $72 \mathrm{~h}$.

SDS-PAGE and western blotting. Whole cells were lysed in RIPA buffer (1\% Triton-100, 0.5\% deoxycholate, $0.2 \%$ sodium dodecyl sulfate, $150 \mathrm{mM}$ sodium chloride, $2 \mathrm{mM}$ ethylene diamine tetra-acetic acid, $10 \mathrm{mM}$ sodium pyrophosphate, $20 \mathrm{mM}$ sodium fluoride) supplemented with a complete protease inhibitor cocktail tablet (Roche Diagnostic, Indianapolis, IN, USA). After cell debris from the protein lysate was removed by centrifugation at $4^{\circ} \mathrm{C}$, protein concentration was measured using a Pierce ${ }^{\mathrm{TM}}$ BCA Protein Assay Kit (Thermo Fisher Scientific, Inc.). Proteins (20 $\mu \mathrm{g}$ per lane) were separated by SDS-PAGE gel, transferred to the nitrocellulose membrane (GE Healthcare) by electrophoresis, then blocked in 5\% non-fat dried milk in PBS plus $0.1 \%$ Tween 20, and incubated with appropriate primary antibodies followed by peroxidase-conjugated secondary antibodies. Protein bands were visualized using ECL detection reagents (GE Healthcare) and photographed using an X-ray film developer.

Cell proliferation and cell attachment assays. A total of $2 \times 10^{4}$ HCC827 control, claudin-7 KD, and KD+b1 cells were seeded in a 6-well plate and the total cell numbers were counted after 2, 4 and 6 days by Trypan Blue exclusion method using a Countess ${ }^{\mathrm{TM}}$ automated cell counter (Invitrogen; Thermo Fisher Scientific, Inc.).

For the cell attachment assay, $2 \times 10^{5}$ HCC 827 control, claudin-7 KD and KD+b1 cells were seeded in a 12-well plate. After $4 \mathrm{~h}$ incubation at $37^{\circ} \mathrm{C}$ in a $5 \% \mathrm{CO}_{2}$ humidified chamber, the culture medium was removed, and each well was washed briefly with PBS buffer to remove unattached cells. Then, the remaining attached cells were trypsinized and mixed with $6 \mu \mathrm{m}$ AlignFlow Plus beads (Molecular Probes; Thermo Fisher Scientific, Inc.) at a concentration of $1 \times 10^{5}$ beads $/ \mathrm{ml}$. Total cell numbers were calculated from the relative ratio of beads to cells using a FACScan flow cytometer.

Wound healing assay. The control, claudin-7 KD, and KD+b1 cells were plated in a 6-well plate until confluent. The cells were then cultured in the serum-free medium for $22 \mathrm{~h}$. After creating a scratch, the serum-containing medium was given, and the gap distance was photographed using an inverted Zeiss light microscope (Carl Zeiss Inc., Thornwood, NY, USA) and analyzed by MetaMorph software (Molecular Devices, LLC, Sunnyvale, CA, USA) at time points of $0,3,6,12$ and $24 \mathrm{~h}$. The closed gap distance per each time point was normalized to its respective initial gap distance at time point 0 per cell line.

In vitro cell invasion assay. A total of $2 \times 10^{5} \mathrm{HCC} 827$ control, $\mathrm{KD}$, and $\mathrm{KD}+\mathrm{b} 1$ cells were suspended and seeded in $500 \mu \mathrm{l}$ of serum-free RPMI-1640 on the membrane of the inner chamber in a 6-well BD Matrigel plate. The outer chamber was also filled with the serum-free medium. After $24 \mathrm{~h}$ serum starvation, the serum-containing culture medium was added in the outer chamber. After $53 \mathrm{~h}$ incubation at $37^{\circ} \mathrm{C}$ in a $5 \% \mathrm{CO}_{2}$ humidified incubator, the membranes from the inner chamber were scrubbed using a medium-wetted cotton swab. Counter cell staining was performed using modified protocols from the Hema-3 Stain kit (Thermo Fisher Scientific, Inc.). In brief, the membranes were first fixed with fixative (Hema-3 Fixative) for 4 min, then stained in red color solution I (Hema-3 Solution I) for $10 \mathrm{~min}$ (cytosol staining), and then stained in blue color solution II (Hema-3 Solution II) for 10 min (nucleus staining). After two brief washes with de-ionized water, the membranes were quickly air-dried and mounted with glycerol on glass slides. Five areas per membrane sample were randomly selected under an inverted light microscope at $200 \mathrm{x}$ magnification using a Zeiss Axiovert S100 microscope (Carl Zeiss Inc.) and photographed using Axiovision 4.6 imaging software (Carl Zeiss Inc.).

Statistical analysis. All experiments were performed at least three times and data were presented as means \pm standard error of means. One- or two-way ANOVA followed by a post-hoc 

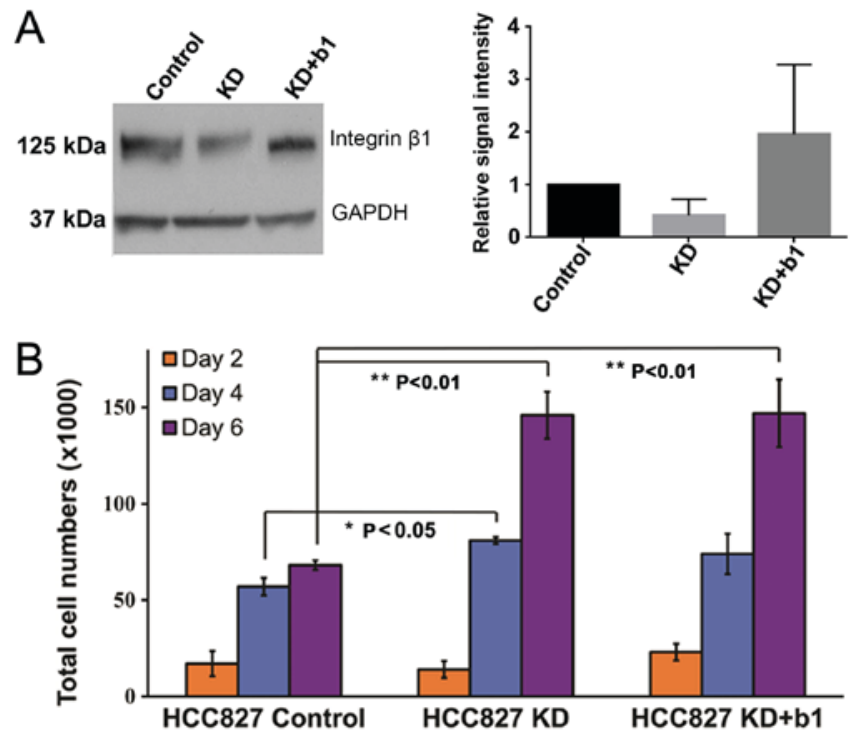

Figure 1. Exogenous integrin $\beta 1$ expression did not reduce hyper-proliferation of claudin-7 KD cells. (A) A representative western blot result shows the integrin $\beta 1$ expression level in control and claudin-7 KD cells (KD), as well as claudin-7 KD cells transfected with integrin $\beta 1 \mathrm{cDNA}$ vector $(\mathrm{KD}+\mathrm{b} 1)$ (Left panel). GAPDH served as a loading control. The right panel shows the densitometry measurements for three independent experiments. There are no statistical significant differences among three groups. (B) A total of $2 \times 10^{4}$ control, $\mathrm{KD}$ and $\mathrm{KD}+\mathrm{b} 1$ cells were seeded in 12 -well plates and the cell numbers were counted for each sample 2, 4, and 6 days following the initial cell culture date. KD+b1 cells did not exhibit any significant changes in cell proliferation rate when compared with Claudin-7 KD cells during the entire cell growth period. The KD and KD+b1 cells exhibited significantly higher proliferating cell numbers when compared to the control cells on day 6 $\left({ }^{* *} \mathrm{P}<0.01\right)$. At least three experiments were performed for statistical analysis. $\mathrm{KD}$, knockdown. ${ }^{*} \mathrm{P}<0.05 ;{ }^{* *} \mathrm{P}<0.01$.

Tukey test was performed in samples from three cell lines using IBM SPSS software (Version 23.0; IBM Corp., Armonk, $\mathrm{NY}, \mathrm{USA}$ ). $\mathrm{P}<0.05$ was considered to indicate a statistically significant difference.

\section{Results}

Ectopic expression of integrin $\beta 1$ did not alter the cell proliferative rate of claudin-7 KD cells. Our previous study showed that HCC827 claudin-7 KD lung cancer cells increased the cell proliferative rate, decreased the expression of a variety of ECM components (including collagen IV and cell adhesion proteins such as integrin $\beta 1$ ), and displayed reduced cell adhesion compared to HCC827 cells with no claudin-7 KD (claudin-7 control cells) (10). Although the reduced cell adhesion and ECM components appeared to accelerate the proliferative rate of the KD cells, supplementing the KD cells with collagen IV improved cell attachment but did not change the cell hyper-proliferative phenotype (10). This suggests that cell adhesion proteins such as integrin $\beta 1$ may be more important in regulating cell adhesion and migration. To investigate whether re-expression of integrin $\beta 1$ could affect the cell proliferative rate of claudin-7 KD cells in a manner comparable to that of control cells, we transfected integrin $\beta 1$ cDNA into the claudin-7 KD cells $(\mathrm{KD}+\mathrm{b} 1)$ as shown in Fig. 1A. The protein expression level of integrin $\beta 1$ in all three cell lines were assayed by Western blotting and
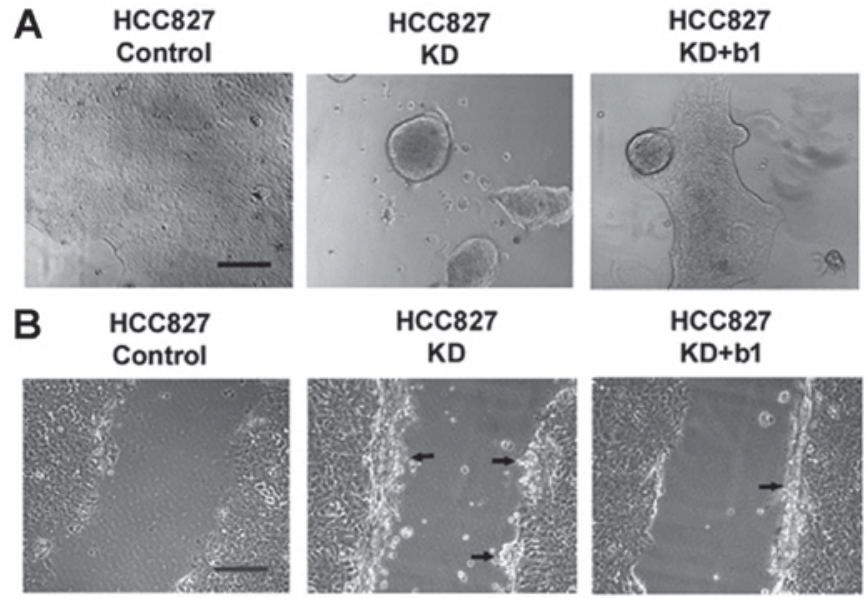

HCC827

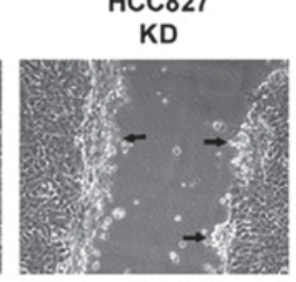

HCC827 KD+b1

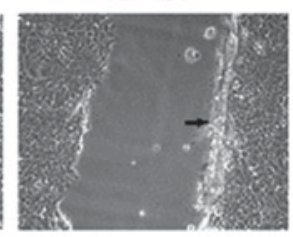

C
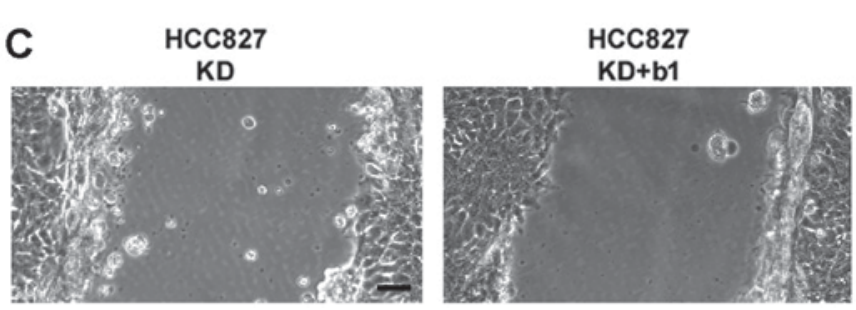

Figure 2. Exogenous integrin $\beta 1$ expression improved cell-matrix adhesion of claudin-7 KD cells. (A) Cells were cultured on glass coverslips. The control cells developed a complete monolayer while all claudin-7 KD cells showed spheroid colonies. KD+b1 cells formed a partial monolayer with a spheroid cell clump indicating incomplete cell spreading. The phase images were photographed at x100 magnification. (B) A scratch was created using a pipet tip on each confluent control, KD and KD+b1 cell monolayer. Compared to the control cells, the KD+b1 cells were attached to the plate fairly well, whereas the claudin-7 KD cells were stripped off along the scratch (shown as black arrows). (C) A higher magnification of $\mathrm{KD}$ and $\mathrm{KD}+\mathrm{b} 1$ cells shown in $\mathrm{B}$ to enlarge the stripped off regions at the scratch site. Scale bar: $10 \mu \mathrm{m}$ for A and $\mathrm{B}, 25 \mu \mathrm{m}$ for $\mathrm{C}$. KD, knockdown.

quantified by densitometry (Fig. 1A). Our results showed that the cell number of KD cells were substantially higher than that of the control cell on day $6(\mathrm{P}<0.01)$ (Fig. 1B), which is consistent with our previous report (10). However, the cell numbers of the KD and KD+b1 cells were not different on day $4\left(8.1 \times 10^{4} \pm 1.7 \times 10^{3}\right.$ vs. $\left.7.4 \times 10^{4} \pm 1.0 \times 10^{4}\right)$ or day 6 $\left(1.5 \times 10^{5} \pm 1.2 \times 10^{4}\right.$ vs. $\left.1.5 \times 10^{5} \pm 1.8 \times 10^{4}\right)$. This result showed that integrin $\beta 1$ expression did not rescue the claudin-7 KD cell hyper-proliferative phenotype, suggesting that integrin $\beta 1$ may not be directly involved in regulating the cell proliferation of claudin-7 KD cells.

Ectopic expression of integrin $\beta 1$ partially recovered the cell adhesion of clauduin-7 KD cells. Although claudin-7 KD cells increased the cell proliferative rate, they exhibited a reduction in cell adhesion and integrin $\beta 1$ expression (10). In addition, integrin $\beta 1$ and claudin-7 formed a protein complex, and they were co-localized at the basolateral membrane of HCC 827 control cells (10). This suggests the possibility of claudin-7 regulation of cell adhesion through integrin $\beta 1$. Therefore, we ectopically expressed integrin $\beta 1$ in the claudin-7 KD cells to examine whether claudin-7 regulated-cell adhesion occurs through integrin $\beta 1$. Similar to the control cells, KD+b1 cells also formed a monolayer on glass coverslips, though a spheroidal clump remained, indicating the incomplete cell 

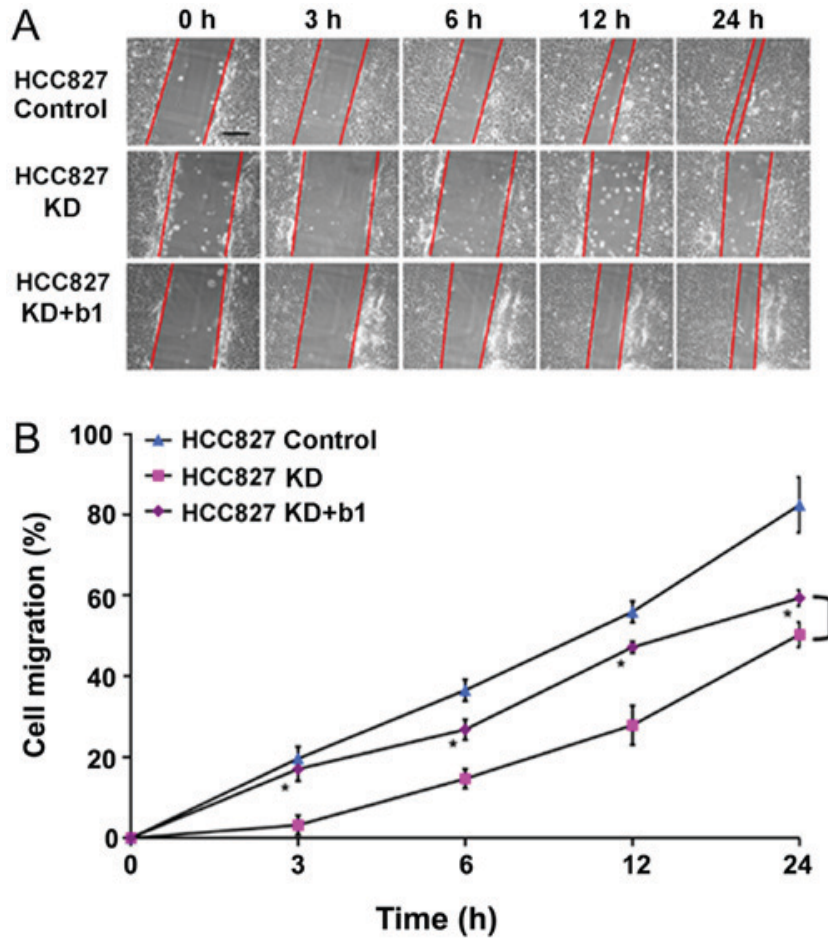

Figure 3. Exogenous integrin $\beta 1$ expression increased the cell migration of claudin-7 KD cells. (A) A panel of representative light phase images of control, $\mathrm{KD}$ and $\mathrm{KD}+\mathrm{b} 1$ cells in wound healing assay plates were photographed at x200 magnification at a specified time point. Scale bar: $10 \mu \mathrm{m}$. (B) Relative cell migration rate on each cell line in wound healing assay. The relative cell migration distance was estimated as the ratio of the closed gap distance at a given time point over initially created gap distance at 0 time point. KD+b1 cells migrated more effectively than claudin-7 KD cells $\left({ }^{*} \mathrm{P}<0.05\right)$. At least three experiments were repeated for statistical analysis. $\mathrm{KD}$, knockdown.

spreading, whereas claudin-7 KD cells displayed spheroidal colonies only, as previously reported (Fig. 2A) (10). Likewise, $\mathrm{KD}+\mathrm{b} 1$ cell layers around the scratch site were less stripped off than the claudin-7 KD cell layers (Fig. 2B, arrows). Fig. 2C show more clearly the stripped off regions at the scratch site of claudin-7 KD and claudin-7 KD +b1 cells. These results demonstrate that ectopic expression of integrin $\beta 1$ partially improved the reduced ability of cell attachment to the dish in the claudin-7 KD cells.

Ectopic expression of integrin $\beta 1$ enhanced cell migration and invasion ability of claudin-7 KD cells. We showed that ectopic expression of integrin $\beta 1$ partially recovered cell adhesion of claudin-7 KD cells, but the effects of claudin-7 via integrin $\beta 1$ on cell motility have not been studied before. Thus, we next examined whether transfection of integrin $\beta 1$ affected the cell migration and invasion of claudin-7 KD cells using wound healing and cell Matrigel invasion assays, respectively. Wound healing assay showed that KD+b1 cells migrated faster than claudin-7 KD cells $(\mathrm{P}<0.05)$, but slower than control cells at all-time points (Fig. 3A and B), indicating that integrin $\beta 1$ increased the KD cell migration caused by claudin-7 knockdown. Additionally, KD+b1 cells invaded more efficiently than claudin-7 KD cells $(\mathrm{P}<0.01)$, but less efficiently than the control cells $(\mathrm{P}<0.05)$ (Fig. 4A and B), indicating that integrin $\beta 1$ transfection also promoted the cell invasiveness of the KD cells.
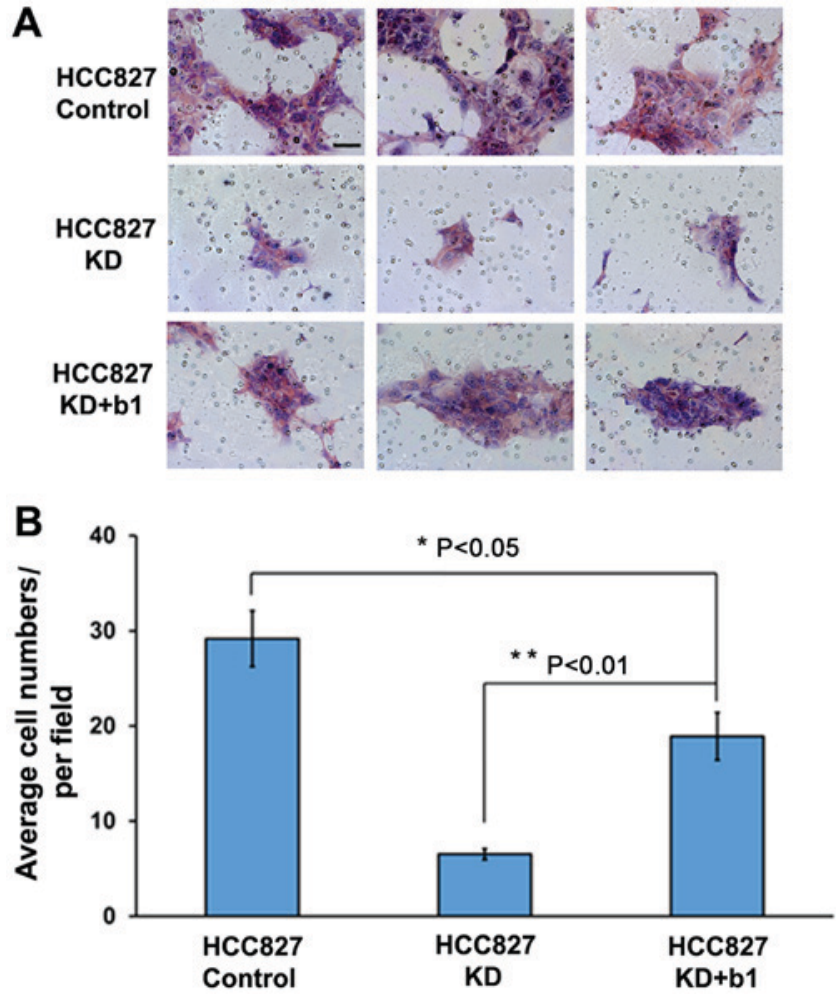

Figure 4. Exogenous integrin $\beta 1$ expression increased the cell invasion ability of claudin-7 KD cells. (A) Cell invasion assay. A panel of representative light phase images of control, KD and KD+b1 cells were photographed at x200 magnification. At least three experiments were performed for statistical analysis shown in B. Scale bar: $20 \mu \mathrm{m}$. (B) The numbers of nucleus stained by blue dye (Hema-3 Solution II) were counted and averaged from 5 randomly chosen areas per cell line from each of three independent experiments. Red-dye (Hema-3 Solution I) staining reflected cytosols. ${ }^{*} \mathrm{P}<0.05 ;{ }^{* *} \mathrm{P}<0.01$. $\mathrm{KD}$, knockdown.

Ectopic expression of integrin $\beta 1$ partially restored defective cell attachment of claudin-7 KD cells. Integrin $\beta 1$ transfection significantly improved the cell adhesion of claudin-7 KD cells, indicating that claudin-7 may result in an increase in cell-matrix attachment via integrin $\beta 1$ at cell-matrix interactions. Thus, we first investigated whether transfection of integrin $\beta 1$ sufficiently improved the cell-matrix attachment of claudin-7 KD cells comparable to the control cells. The number of KD+b1 cells that remained attached was significantly higher than that of claudin-7 KD cells $(\mathrm{P}<0.05)$, but significantly lower than that of control cells $(\mathrm{P}<0.01)$ in the cell attachment assay (Fig. 5A). This indicates that integrin $\beta 1$ transfection partially restores the defect in the cell attachment of claudin-7 KD cell.

In order to fully restore the defective KD cell attachment, we transiently transfected claudin-7 cDNA containing Myc-tag into the KD cells to generate KD+cldn7 cells. The cell attachment results showed that the number of KD+cldn7 cells that remained attached was significantly higher than that of control cells $(\mathrm{P}<0.01)$ or claudin-7 KD cells $(\mathrm{P}<0.01)$ (Fig. 5B), indicating that overexpression of claudin-7 strengthened the cell-matrix attachment of the KD cells far better. Western blot analysis confirmed the ectopic expression of integrin $\beta 1$ or claudin-7 in KD cells (Fig. 5C). Although integrin $\beta 1$ expression moderately increased the level of phospho-FAK in KD cells compared to that of the control cells, claudin-7 
A

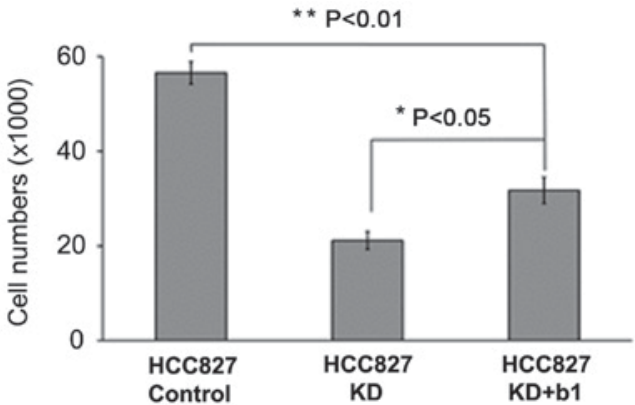

B

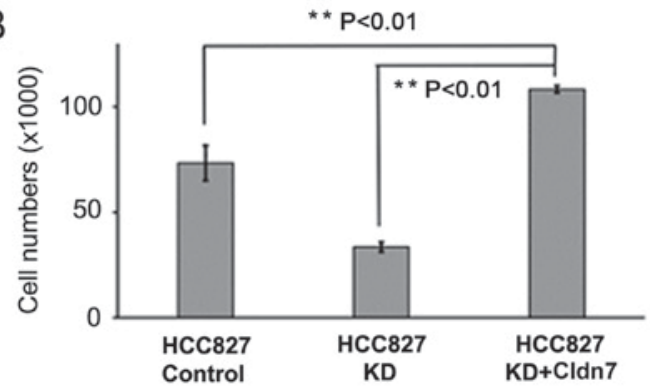

C

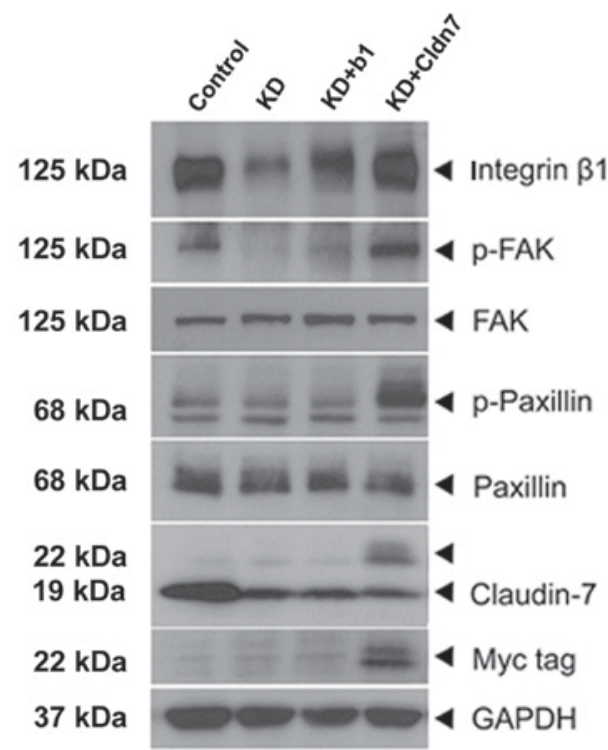

Figure 5. Both integrin $\beta 1$ and claudin-7 improved the cell attachment capability of claudin-7 KD cells to different degrees. (A) Cell attachment assay testing the effect of integrin $\beta 1$ overexpression in KD cells. A total of $2 \times 10^{5}$ cells per cell line were seeded in a 12-well plate and incubated for $4 \mathrm{~h}$ at $37^{\circ} \mathrm{C}$ in a $5 \% \mathrm{CO}_{2}$ humidified incubator. After unattached cells were washed briefly with PBS buffer, the remaining attached cells were trypsinized and counted for evaluation. $\mathrm{KD}+\mathrm{b} 1$ cells showed significantly more attached cells than claudin-7 KD cells ( $\mathrm{P}<0.05)$, but significantly fewer than control cells $\left({ }^{* *} \mathrm{P}<0.01\right)$. At least three experiments were repeated for statistical analysis. (B) Cell attachment assay testing the effect of claudin-7 overexpression in claudin-7 KD cells. A total of $2 \times 10^{5}$ control, $\mathrm{KD}$, and $\mathrm{KD}+\mathrm{cldn} 7$ were seeded in a 12-well plate and incubated for $4 \mathrm{~h}$ at $37^{\circ} \mathrm{C}$ in a $5 \% \mathrm{CO}_{2}$ incubator. Unattached cells were washed briefly using PBS buffer, and remaining attached cells were trypsinized and counted for evaluation. KD+cldn7 cells increased the attached cell numbers significantly more than both KD cells $\left({ }^{* *} \mathrm{P}<0.01\right)$ and control cells $\left({ }^{* *} \mathrm{P}<0.01\right)$. At least three repetitive experiments were performed for statistical analysis. (C) A representative western blot result on protein expression levels of focal adhesion proteins in control, claudin-7 KD, KD+b1, and KD+cldn7 cells. KD+b1 cells increased the expression levels of integrin $\beta 1$ and phospho-FAK, but not phospho-Paxillin when compared to KD cells. KD+cldn7 cells increased protein expression levels of integrin $\beta 1$, phospho-FAK, and phospho-Paxillin. KD+cldn7 cells showed both an exogenous claudin-7 band at $22 \mathrm{kDa}$ with Myc-tag and endogenous claudin-7 expression at $19 \mathrm{kDa}$. GAPDH served as a loading control. KD, knockdown. ${ }^{*} \mathrm{P}<0.05 ;{ }^{* * *} \mathrm{P}<0.01$. overexpression greatly elevated the expression levels of phospho-FAK, phospho-Paxillin as well as integrin $\beta 1$ in KD+cldn7 cells when compared to control cells (Fig. 5C). This result indicates that both integrin $\beta 1$ and claudin-7 synergistically support cell attachment, although claudin-7 appears to have a greater effect than integrin $\beta 1$.

Taken together, these results suggest that ectopic expression of integrin $\beta 1$ in claudin-7 KD cells partially recovered the control cell adhesion, migration and invasion, but not cell proliferation.

\section{Discussion}

In this study, we noticed that ectopic expression of integrin $\beta 1$ in claudin-7 KD cells did not lead to claudin-7 expression, but claudin-7 overexpression in the KD cells sufficiently revived integrin $\beta 1$ expression. Our previous study also demonstrated that claudin-7 and integrin $\beta 1$ formed a protein complex co-localized at the basolateral membrane of HCC 827 control cells (10). These results suggest that claudin-7 and integrin $\beta 1$ may co-operate with each other, although claudin-7 seems to have a greater ability to control cellular phenotypes. For example, ectopic expression of integrin $\beta 1$ did not change the hyper-proliferative rate of claudin-7 KD cells. However, claudin-7 overexpression in KD cells reduced the cell proliferation rate (10). This indicates that claudin-7 may enable integrin $\beta 1$ at the basolateral membrane to properly receive signaling from the ECM to control lung cancer cell proliferation. This does not align with some of the reports that integrin $\beta 1$ signaling regulates cell proliferation and survival (14). For example, deletion of integrin $\beta 1$ reduces cell proliferation of mammary gland at the cellular level $(15,16)$. At the organ level, integrin $\beta 1$ knockout mice die before birth (15) probably due to the absence of the integrin $\beta 1$ signaling. Interestingly, our claudin-7 KD cells suppressed integrin $\beta 1$ expression but accelerated cell proliferation (10). It is possible that other $\beta$ integrins could compensate for the lack of integrin $\beta 1$ signaling to promote cell proliferation. In a breast cancer cell study, integrin $\beta 3$ signaling is activated to increase cell proliferation and invasion when integrin $\beta 1$ is suppressed (17). Future investigation is thus warranted to clarify the compensatory effect of other $\beta$ integrins in claudin- $7 \mathrm{KD}$ cells.

Claudin-7 also synergistically collaborates with integrin $\beta 1$ to control cell adhesion, migration, invasion and attachment. We demonstrated that KD+b1 cells have improved cell-matrix adhesion when compared to claudin-7 KD cells (Fig. 2), indicating that ectopic expression of integrin $\beta 1$ supports the role of claudin-7 in cell-matrix adhesion through the basolateral membrane of KD cells. However, KD+b1 cells still showed a reduction in the rate of the cell migration when compared to control cells. Cell migration requires proper cell-matrix adhesion: Focal adhesion, which primarily consists of integrin clustering through cell-matrix interface, maintains an extended actin cytoskeletal protrusion in the direction of cell migration, and it is further stabilized by nascent adhesion along with lamellipodia at the leading cell edge $(18,19)$. The consequentially skewed cell shape accumulates contractile force, which eventually drives cell migration when rear cell adhesion is released $(18,19)$. When claudin-7 is low in quantity, focal adhesion through the basolateral membrane may not be 
properly established, inhibiting the subsequent process of cell migration. This suggests that claudin-7 cooperatively regulates cell migration via integrin $\beta 1$. However, our study also does not rule out the possibility that claudin-7 and integrin $\beta 1$ act independently.

Similar to the cell migration process, cell-matrix adhesion is also essential in cell invasiveness. Integrins form focal adhesion complexes, which results in the creation of actin cytoskeletal protrusion as an invadopodia precursor (20). In addition, integrin $\beta 1$ recruits integrin-linked kinase (ILK) that transforms the invadopodia precursor into adhesion ring-containing matured invadopodia (21), which allows the extension of the actin cytoskeletal protrusions and activates ECM degradation activity (20-22). The low amount of claudin-7 may reduce the number of focal adhesion complexes and prevent further processes of cell invasiveness. Although ectopic expression of integrin $\beta 1$ in KD+b1 cells may increase the numbers of integrin clustering, the low quantity of claudin-7 in the KD+b1 cells may not effectively anchor the integrins $\beta 1$ through the basolateral membrane and thus make it unable to fully recover the intensity of focal adhesion and degradation enzyme activity as it does in the control cells.

Likewise, cell-matrix adhesion is crucial in cell-matrix attachment. Claudin-7 overexpression in claudin-7 KD cells $(\mathrm{KD}+\mathrm{cldn} 7)$ recovers the cell attachment ability at a far higher level than that of control cells. The increased cell attachment in claudin-7 overexpression is due to the excessive level of focal adhesion proteins confirmed by Western blot results, suggesting that the claudin-7 overexpression at the basolateral membrane of $\mathrm{KD}+\mathrm{cldn} 7$ cells may bind stronger to the target ECM surface than that of control cells. We were unable to test the metastatic possibility in vivo due to the intrinsically poor metastatic potential of HCC827 cell lines (23). Future study should include more lung cancer cell lines and the in vivo test.

It is interesting to notice that the ectopically expressed claudin-7 leads to a significant increase in cell numbers and strong activation of integrin signaling although its expression level is not as high as the endogenous claudin-7 level in control cells (Fig. 5B and C). One possibility could be due to the claudin-7 phosphorylation level. We know that claudin-7 is a phosphorylated membrane protein (24). It is seen that the ectopically expressed claudin-7 has a higher phosphorylation level compared to the endogenous claudin-7. It is possible that the phosphorylated claudin-7 tends to be membrane bound while non-phosphorylated claudin-7 largely stays in the cytoplasm. It could be the phosphorylated claudin-7 that plays a major role in regulating integrin $\beta 1, \mathrm{p}-\mathrm{FAK}$, and p-Paxillin expression. Future work is needed to investigate the differential roles of phosphorylated and non-phosphorylated claudin-7.

In addition, claudin-7 proteins may serve as anchoring domains to recruit other cell adhesion proteins at cell membrane, as previously shown for integrin $\beta 1$ (10) as well as other cell adhesion proteins, including CD44 and EpCAM $(25,26)$, all of which could contribute to the overall cell-matrix adhesion of lung cancer cells. This may explain, in part, why transfection of integrin $\beta 1$ in claudin-7 KD cells did not fully recover the cell-matrix adhesion, migration, invasion, and attachment.
The ability of claudin-7 interaction with integrin $\beta 1$ to establish cell-matrix adhesion appears to be important to understand the molecular mechanism of Human Immunodeficiency Virus (HIV) infection. It has been reported that HIV infected CD4(-) T cell subpopulation when claudin-7 gene was introduced to the HIV particles in vitro (27). However, it remained unclear whether surface ligands in the CD4(-) T cells could interact with claudin-7 on the viral particles. Recently, it has also been reported that peripheral blood lymphocytes from normal patients show the high-level integrin $\beta 1$ (CD29) surface marker in $66 \%$ of CD4(-) $\mathrm{T}$ cell subpopulation (28). This suggests that claudin-7 proteins in the HIV-1 viral coat may interact with integrins $\beta 1$ (CD29) present in the host CD4(-) T cells, which could establish mutual membrane adhesion that allows mediation of the membrane fusion process for entry of the HIV particles to CD4(-) T cells.

In this report, we conclude that claudin-7 functions as a cell adhesion protein that modulates cell-matrix adhesion and regulates cellular process, including lung cancer cell migration, invasion, and attachment.

\section{Acknowledgements}

The authors would like to thank Mr. Rodney Tatum, Mrs. Beverly G. Jeansonne, and Ms. Christi Boykin for their technical assistance and Dr Kvin Lertpiriyapong (all Brody School of Medicine at East Carolina University) for providing constructive comments for this manuscript.

\section{Funding}

This study was supported by National Institute of Health (Grant no. HL085752).

\section{Availability of data and materials}

The datasets used/analyzed in this study are available from the corresponding author upon reasonable request.

\section{Authors' contributions}

DHK performed experiments, collected and analyzed data as well as wrote the manuscript. QL conceptualized the study, read and edited the manuscript. YHC designed the study, interpreted data, and finalized the manuscript. All authors read and approved the final manuscript as submitted.

\section{Ethics approval and consent to participate}

All experimental procedures were approved by the Brody School of Medicine at East Carolina University (Greenville, NC, USA).

\section{Patient consent for publication}

Not applicable.

\section{Competing interests}

The authors declare that they have no competing interests. 


\section{References}

1. Siegel R, Naishadham D and Jemal A: Cancer statistics, 2012. CA Cancer J Clin 62: 10-29, 2012.

2. Soini Y: Tight junctions in lung cancer and lung metastasis: A review. Int J Clin Exp Pathol 5: 126-136, 2012.

3. Chang EH, Pezzulo AA and Zabner J: Do cell junction protein mutations cause an airway phenotype in mice or humans? Am J Respir Cell Mol Biol 45: 202-220, 2011.

4. Gunzel D and Yu AS: Claudins and the modulation of tight junction permeability. Physiol Rev 93: 525-569, 2013.

5. Philip R, Heiler S, Mu W, Büchler MW, Zöller M and Thuma F: Claudin-7 promotes the epithelial-mesenchymal transition in human colorectal cancer. Oncotarget 6: 2046-2063, 2015.

6. Bhat AA, Pope JL, Smith JJ, Ahmad R, Chen X, Washington MK, Beauchamp RD, Singh AB and Dhawan P: Claudin-7 expression induces mesenchymal to epithelial transformation (MET) to inhibit colon tumorigenesis. Oncogene 34: 4570-4580, 2015.

7. Morin PJ: Claudin proteins in human cancer: Promising new targets for diagnosis and therapy. Cancer Res 65: 9603-9606, 2005.

8. Escudero-Esparza A, Jiang WG and Martin TA: Claudin-5 is involved in breast cancer cell motility through the N-WASP and ROCK signalling pathways. J Exp Clin Cancer Res 31: 43, 2012.

9. Yamamoto T, Oshima T, Yoshihara K, Yamanaka S, Nishii T, Arai H, Inui K, Kaneko T, Nozawa A, Woo T, et al: Reduced expression of claudin-7 is associated with poor outcome in non-small cell lung cancer. Oncol Lett 1: 501-505, 2010.

10. Lu Z, Kim DH, Fan J, Lu Q, Verbanac K, Ding L, Renegar R and Chen YH: A non-tight junction function of claudin-7-Interaction with integrin signaling in suppressing lung cancer cell proliferation and detachment. Mol Cancer 14: 120, 2015.

11. Poon CE, Madawala RJ, Day ML and Murphy CR: Claudin 7 is reduced in uterine epithelial cells during early pregnancy in the rat. Histochem Cell Biol 139: 583-593, 2013.

12. Gonzalez-Mariscal L, Namorado Mdel C, Martin D, Sierra G and Reyes JL: The tight junction proteins claudin-7 and -8 display a different subcellular localization at Henle's loops and collecting ducts of rabbit kidney. Nephrol Dial Transplant 21: 2391-2398, 2006.

13. Blackman B, Russell T, Nordeen SK, Medina D and Neville MC: Claudin 7 expression and localization in the normal murine mammary gland and murine mammary tumors. Breast Cancer Res 7: R248-R255, 2005

14. Srichai MB and Zent R: Integrin structure and function. In: Cell-Extracellular Matrix Interactions in Cancer. Zent $R$ and Pozzi A (eds). Springer New York, New York, NY, pp19-41, 2010.

15. Liu S, Xu SW, Blumbach K, Eastwood M, Denton CP, Eckes B Krieg T, Abraham DJ and Leask A: Expression of integrin beta1 by fibroblasts is required for tissue repair in vivo. J Cell Sci 123: 3674-3682, 2010.

16. Li N, Zhang Y, Naylor MJ, Schatzmann F, Maurer F, Wintermantel T, Schuetz G, Mueller U, Streuli $\mathrm{CH}$ and Hynes NE: Betal integrins regulate mammary gland proliferation and maintain the integrity of mammary alveoli. EMBO J 24: 1942-1953, 2005.
17. Parvani JG, Galliher-Beckley AJ, Schiemann BJ and Schiemann WP: Targeted inactivation of $\beta 1$ integrin induces $\beta 3$ integrin switching, which drives breast cancer metastasis by TGF- $\beta$. Mol Biol Cell 24: 3449-3459, 2013.

18. Bravo-Cordero JJ, Magalhaes MA, Eddy RJ, Hodgson L and Condeelis J: Functions of cofilin in cell locomotion and invasion. Nat Rev Mol Cell Biol 14: 405-415, 2013.

19. Bouvard D, Pouwels J, De Franceschi N and Ivaska J: Integrin inactivators: Balancing cellular functions in vitro and in vivo. Nat Rev Mol Cell Biol 14: 430-442, 2013.

20. Murphy DA and Courtneidge SA: The 'ins' and 'outs' of podosomes and invadopodia: Characteristics, formation and function. Nat Rev Mol Cell Biol 12: 413-426, 2011.

21. Branch KM, Hoshino D and Weaver AM: Adhesion rings surround invadopodia and promote maturation. Biol Open 1: 711-722, 2012.

22. Magalhaes MA, Larson DR, Mader CC, Bravo-Cordero JJ, Gil-Henn H, Oser M, Chen X, Koleske AJ and Condeelis J: Cortactin phosphorylation regulates cell invasion through a pH-dependent pathway. J Cell Biol 195: 903-920, 2011.

23. Furugaki K, Moriya Y, Iwai T, Yorozu K, Yanagisawa M, Kondoh K, Fujimoto-Ohuchi K and Mori K: Erlotinib inhibits osteolytic bone invasion of human non-small-cell lung cancer cell line NCI-H292. Clin Exp Metastasis 28: 649-659, 2011.

24. Hoggard J, Fan J, Lu Z, Lu Q, Sutton L and Chen YH: Claudin-7 increases chemosensitivity to cisplatin through the upregulation of caspase pathway in human NCI-H522 lung cancer cells. Cancer Sci 104: 611-618, 2013

25. Kuhn S, Koch M, Nübel T, Ladwein M, Antolovic D, Klingbeil P, Hildebrand D, Moldenhauer G, Langbein L, Franke WW, et al: A complex of EpCAM, claudin-7, CD44 variant isoforms, and tetraspanins promotes colorectal cancer progression. Mol Cancer Res 5: 553-567, 2007.

26. Ladwein M, Pape UF, Schmidt DS, Schnölzer M, Fiedler S, Langbein L, Franke WW, Moldenhauer G and Zöller M: The cell-cell adhesion molecule EpCAM interacts directly with the tight junction protein claudin-7. Exp Cell Res 309: 345-357, 2005.

27. Zheng J, Xie Y, Campbell R, Song J, Massachi S, Razi M, Chiu R, Berenson J, Yang OO, Chen IS and Pang S: Involvement of claudin-7 in HIV infection of CD4(-) cells. Retrovirology 2: 79, 2005.

28. Leblond V, Legendre C, Gras G, Dereuddre-Bosquet N, Lafuma C and Dormont D: Quantitative study of betal-integrin expression and fibronectin interaction profile of $\mathrm{T}$ lymphocytes in vitro infected with HIV. AIDS Res Hum Retroviruses 16: $423-433,2000$

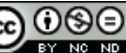

This work is licensed under a Creative Commons Attribution-NonCommercial-NoDerivatives 4.0 International (CC BY-NC-ND 4.0) License. 